\title{
Modifying Monolayer Behaviour by Incorporating Subphase Additives and Improving Langmuir Blodgett Thin Film Deposition on Optical Fibres.
}

\author{
Matthew Partridge ${ }^{1,3}$, Rebecca Wong ${ }^{3}$, Mike Collins ${ }^{2}$, Stephen W James ${ }^{3}$, Frank Davis ${ }^{1 *}$, Ralph P \\ Tatam $^{3}$, Séamus P J Higson ${ }^{1}$ \\ ${ }^{1}$ Cranfield Health, Cranfield University \\ ${ }^{2}$ Leksing Ltd, Colworth Science Park, Bedfordshire, \\ ${ }^{3}$ Department of Engineering Photonics, School of Engineering, Cranfield University \\ *Dr Frank Davis: phone +44(0)1234 758332; e-mail: f.davis@ cranfield.ac.uk
}

\begin{abstract}
Experiments showing the possibility of modifying the behaviour of calix[4]resorcinarene monolayers at the air-water interface and optimising the deposition of multilayer coatings onto optical fibres are presented. The nature of the subphase is fundamental to the behaviour of monolayers and their utility in coating and sensing applications. Here we show initial studies exploring the modification of the calix[4]resorcinarene monolayer water interaction through the introduction of dipole altering alcohol additives to the aqueous subphase. We explored the effect of this modification for three small alcohols. The resulting isotherms of the materials showed a reduction in the surface pressure and area per molecule required in order for the monolayer to reach its point of collapse. Incorporation of alcohols shifted the point of collapse, leading to the application of ethanol being successful in improving the transfer of material via LangmuirBlodgett coating onto optical fibres at lower pressures. This method may prove useful in allowing greater control over future sensor surface coatings.
\end{abstract}

\section{Keywords : monolayers; multilayers; coatings; optical properties}




\section{Introduction}

Studies on Langmuir monolayers have encompassed a wide range of effects and properties of the monolayer materials. Thanks to a number of new developments in microscopy [1], spectroscopy [2] and range of other analytical techniques, the field has widened to produce a number of models and theories on the way monolayers behave and how they react [3]. One area that has received attention is the modification of the interaction between the monolayer material and the subphase, on which it is spread. Although much work on incorporation of salts into subphases and their effects on monolayers has been published, the use of uncharged polar molecules has been less studied.

When an amphiphilic substance is spread on the surface of water, it will form a disperse monolayer, orientated so that the hydrophilic head is immersed in or on the surface of the water, with the insoluble hydrophobic tail orientated away from the water. The solubility of the head group is known to be determined by, amongst other factors, the dipole moment of any groups it contains [4]. The difference in dipole moments between the monolayer material and the subphase determines the degree of attraction/solubility [5]. This interaction has been explored in a variety of ways, including characterisation using surface potential measurements, which has facilitated the measurement of the dipole interaction [6].

When the monolayer is compressed, the area per molecule decreases and the surface pressure increases until in many (but not all) cases the molecules form a stable condensed monolayer. At this point, the surface pressure increases rapidly with further decrease in area. Eventually the monolayer collapses, typically causing a sudden drop in surface pressure [7] or the development of a plateau in the rate of surface pressure increase [8]. This collapse is governed by the forces applied by the surrounding molecules in overcoming the dipole-dipole interaction of the monolayer material with either the surrounding monolayer molecules or the aqueous subphase [8]. Both thermodynamic and kinetic effects can be factors in determining the collapse pressure since a condensed monolayer is often not in a state of thermodynamic stability.

In Langmuir-Blodgett (LB) coatings, the packing of molecules must be sufficient to enable the transfer of the material to a solid surface. Frequently this means that the monolayer is compressed to a surface-pressure close to the point of collapse to ensure that the material is nearing the force required to lift off the subphase and on to the substrate. The point at which the highest coating ratio is achieved is fixed for a given material and so the density of the coating is similarly influenced by this property [9].

There has been much previous work describing the effects of adding a variety of materials to the subphase, for instance the incorporation of metal salts beneath monolayers of carboxylic acids to give monolayers of metal carboxylates [4]. Changing the temperature of the subphase can also have major effects on the isotherms and deposition of many materials [4]. Through modification of the dipole-potential of the subphase, it is suggested that the surface pressure and area at collapse (a direct measure of the packing of the monolayer) can be altered. Much previous work on subphase doping and modification has focused on material specific effects such as the addition of ions on carboxylic acid monolayers $[10,11]$. However other workers have investigated the 
effect of alcohol modification of subphases. A number of monoalkyl and dialkyl glycerides were studied on subphases containing methanol or ethanol [12-14]. They found that incorporating alcohols in the subphase influenced the isotherms, for example incorporation of ethanol led to decreases in the collapse pressure, a reduction in surface area and decreases in liquid-condensed to solid phase transition pressure [12]. Similar results were also found for fatty acid monolayers [15]. Addition of ethanol was also thought to lead to formation of a more rigid monolayer $[12,14,15]$. Similar results showing reductions in surface area and elasticity were noted for phospholipid layers on ethanol containing subphases as opposed to water [16].

Previously we have investigated a range of calix[4]resorcinarenes as monolayers at the air waterinterface [17] as well as investigating their deposition onto optical fibres for use as sensors for volatile organic compounds [18]. Within this work we describe how the monolayer behaviour and deposition characteristics of two calix[4]resorcinarenes are affected by the presence of alcohols, especially ethanol, in the subphase.

The aim of this work was to determine whether such additives could be used to modify the coating behaviour of LB materials and to investigate whether optical fibres could be used to characterise the deposition of monolayers. The ability to affect the dipping characteristics would be beneficial to many sensor-coating applications.

\section{Materials and methods}

All experiments were performed using a Nima alternate layer Langmuir trough (Type: 622) filled with distilled water (18 MOhms cm resistivity). For experiments using doped subphases, the distilled water was combined with methanol (99.8\%, Fisher), ethanol (99.5\%, Sigma) or propan1-ol (99.7\%, Sigma). All surface pressure measurements were recorded with a Wilhelmy plate system using a filter paper plate. Between each series of experiments, the trough was drained and then wiped clean with acetone and isopropyl alcohol soaked tissues to remove any contaminants.

The structure of the resorcinarenes used is shown in Figure 1, both of these are amphiphilic and will orientate in a similar fashion with their hydrocarbon chains held away from the subphase, as previously reported [17]. The primary route chosen to modify the monolayer-water interaction was to interfere with the potential of the water to hydrogen bond, both to itself and to any monolayer material. It is well understood that introduction of alcohols to water disrupts the hydrogen bonding and will lower its boiling point. This disruption is due to the interference of the hydrocarbon tails of these simple alcohols. This effect is clearest in the influence that alcohols have on the surface tension of water; concentrations as low as $10 \%$ can cause a $20 \mathrm{mN} \mathrm{m}^{-1}$ reduction in surface tension [19].

Monolayers of Res-1 were prepared by spreading $50 \mu \mathrm{l}$ of a $1 \mathrm{mg} \mathrm{ml}^{-1}$ solution on the surface of a subphase, which consisted of either deionized water or a solution of methanol, ethanol or propan1-ol. After introduction of the each material to the subphase, it was left to spread for 30 mins.

Isotherms were defined by compressing the monolayer at $50 \mathrm{~cm}^{2} \mathrm{~s}^{-1}$. It is well known that varying the compression speed can have a major effect on isotherms and since our interest was to determine the effect of subphase doping on the monolayer behaviour, a single compression speed 
was used for all experiments for consistency. Repeated spreading and compression of fresh films of the same materials demonstrated that the relative standard deviation of the surface area per molecule was $<3 \%$.

To investigate the influence of the presence of alcohol on the LB coating, LB films were deposited onto a silica capillary, a silica slide and a silica optical fibre. The chloroform solutions were applied to the surface of the trough, allowed to spread for 30 mins and compressed to the appropriate surface pressure (initially $20 \mathrm{mN} \mathrm{m}^{-1}$ ). Once at pressure, each film was left for 1 hour in order to ensure that it had stabilised. In all cases stable monolayers were obtained as shown by minimal change of surface area with time after a few minutes. After the stabilisation time, the coating experiments were carried out.

The initial experiments used a single silica glass capillary tube, which had been sealed at both ends. Deposition was also successfully achieved on glass microscope slides. However silica capillaries were used since they are closer in shape to the optical fibres to be used later. The capillary tube (diameter $2 \mathrm{~mm}$ ) was mounted on to the LB dipper in a vertical position relative to the surface of the water to allow for a $20 \mathrm{~mm}$ long section in the middle of the tube to be coated; the tube had a total coated surface area of $1.25 \mathrm{~cm}^{2}$ per stroke. Prior to coating and in between each coating experiment, the capillary tube was cleaned ultrasonically in deionised water and $50 \%$ ethanol for 10 minutes, then wiped repeatedly with clean room filter paper soaked in chloroform. For each dip, the capillary tube started in the submerged position and was moved through the monolayer at a rate of $15 \mathrm{~mm} \mathrm{~min}^{-1}$. After the deposition of a series of layers at the required pressure, the capillary was immersed in the subphase, the pressure increased for the next experiment (at $25 \mathrm{mN} \mathrm{m}^{-1}$ ) and again allowed to stabilise, as shown by constant surface pressure and minimal change in surface area. Between each run of experiments the trough was emptied and cleaned according to the standard method.

The process used for coating optical fibres in the trough is similar to that described for the capillary tube; the optical fibre was mounted on a cross bar that held the fibre with its long axis at 90 degrees to the surface of the water. The fibre was held by two small screws, approximately 6 $\mathrm{cm}$ apart, mounted on the cross bar. The cross bar was then attached to a Nima alternate layer dipping rig which is able to move the fibre straight up and down through the monolayer's surface (figure 2). The proximal and distal ends of the fibre were connected to a tungsten-halogen bulb light source and spectrophotometer respectively, which were used to facilitate the monitoring of the transmission spectrum throughout the coating experiment. Once mounted, the fibres were left in place for several coating experiments and were wiped with acetone and isopropyl alcohol between each coating run. Because of the small diameter of the optical fibres $(125 \mu \mathrm{m})$, accurate deposition ratios cannot be obtained for each stroke. 


\section{Results and discussion}

\subsection{Effect of alcohols on monolayer behaviour.}

Initial work studied the effects of different concentrations of ethanol $(100,200$ and $400 \mathrm{mM})$ on the isotherms. However the lowest concentration was found to have very small effects, with the difference in isotherms on water and ethanol solution being less than the repeatability error of the isotherms themselves. The highest concentration also led to problems due to the reduction in subphase surface tension and evaporation of the alcohol from the subphase so $200 \mathrm{mM}$ was chosen as the most convenient concentration to use.

Figure 3 shows the isotherms of calix[4]resorcarene on water and $200 \mathrm{mM}$ alcohol subphases. The classical shape of the isotherms with a steep rise in pressure as the surface area decreases, combined with the surface areas obtained indicate formation of ordered monolayers. At low pressure $\left(<15 \mathrm{mN} \mathrm{m}^{-1}\right)$, the water, methanol and ethanol subphases have little influence on the isotherm, with the addition of the alcohols leading to small increases in surface area per molecule. A somewhat larger increase in surface area per molecule was seen for propan-1-ol. The water and methanol subphase isotherm become almost identical as the pressure increased, until $43 \mathrm{mN} \mathrm{m}^{-1}$ where the first sign of collapse appears, with the monolayer on the methanol subphase collapsing more quickly. Final collapse occurred at $50 \mathrm{mN} \mathrm{m}^{-1}$ on methanol and $53 \mathrm{mN} \mathrm{m}^{-1}$ on water. The monolayer on the ethanol subphase showed a smaller surface area than that observed on water at higher pressures $\left(>15 \mathrm{mN} \mathrm{m}^{-1}\right)$, with a reduction in the gradient of the isotherm at $35 \mathrm{mN} \mathrm{m}^{-1}$ and final collapse at $45 \mathrm{mN} \mathrm{m}^{-1}$. Post collapse, the monolayers on the subphases showed a steady plateau in surface pressure for all subphases apart from that containing propan-1-ol, which again displayed the lowest surface area and collapse pressure of the group. The longer the alcohol hydrocarbon chain, the lower the plateau surface pressure. For a propan-1-ol containing subphase the monolayer collapsed at approximately $30 \mathrm{mN} \mathrm{m}^{-1}$ with a slow increase in pressure after this. This demonstrates that as the alcohols become longer, their effect on the monolayer became more prominent. This was especially true for the longest alcohol, propan-1-ol which in itself may display significant surface activity and disrupt the monolayer. Other workers have also reported that ethanol shows some surfactant like behaviour and can be incorporated into monolayers [12$14,16]$. As a result of these experiments, it was decided that work would focus on comparing the water and $200 \mathrm{mM}$ ethanol subphases to determine the effect on LB deposition.

Within figure 4 we show some typical examples of deposition profiles for the resorcinarenes. As shown in figure 4 , Res-1 shows poor coating at surface pressures lower than $35 \mathrm{mN} \mathrm{m}^{-1}$. This poor coating is typified by unevenly spaced area changes with each successive layer and sudden gains in surface pressure as the coating detaches from the substrate. The investigation presented here goes further than previously reported $[17,18]$ and studied lower surface pressures $\left(20-30 \mathrm{mN} \mathrm{m}^{-1}\right)$, which appears to show both coated monolayer degradation with sudden jumps in surface area and more notably the formation of X-type coating on the down stroke only at $20 \mathrm{mN} \mathrm{m}^{-1}$, which has not been previous observed. However high transfer ratios, an indicator of good deposition, 
appeared to occur only on the first down stroke with the transfer ratios dropping well below 1 in subsequent dips. When the pressure was increased to $25 \mathrm{mN} \mathrm{m}^{-1}$ there was initially transfer for the first cycle but, as shown in figure 4, subsequent cycles become irregular with "peeling off" of the monolayer occurring on down strokes and sudden changes in surface area on upstrokes. Similar behaviour occurred at 30 and $35 \mathrm{mN} \mathrm{m}^{-1}$. At $37.5 \mathrm{mN} \mathrm{m}^{-1}$ a regular Z-type deposition occurs (examples of deposition traces are shown in Figure 4). When the surface pressure was increased to $40 \mathrm{mN} \mathrm{m}^{-1}$ Y-type deposition occurred. For the purposes of sensor preparation however, further work will focus on the higher surface pressure range as this is most likely to give the greatest density of coating which is often desirable to many sensing applications.

Given the total coatable surface area of $1.25 \mathrm{~cm}^{2}\left(+/-0.01 \mathrm{~cm}^{2}\right)$, it is interesting to note that, from the area loss, we can calculate that at $37.5 \mathrm{mN} \mathrm{m}^{-1}$ there was approximately a $200 \%$ transfer ratio of material to the surface, indicating that bilayers were being deposited. This continued for subsequent layers deposited at this surface pressure. It has been observed previously that at higher pressure, calix monolayers take time to find their equilibrium position/state. The areas per molecule and general isotherm shape indicate that monolayers are being formed on the water surface. It appears that on transfer to the solid substrate, the actual dipping process leads to localised changes in the monolayer, leading to collapse, possibly to form a bilayer which then transfers onto the glass substrate. When transfer is ended the monolayer rapidly stabilises. This means that the initial area loss may be a result of a combination of deposition and reaching equilibrium. An example of this is shown in figure 4 where at $40 \mathrm{mN} \mathrm{m}^{-1}$ the initial deposition appeared to be more than a monolayer but providing enough time was left then good reproducible Y-deposition with a transfer ratio of approximately $100 \%$ became standard. Multilayers in excess of 100 layers thick could be deposited by this method.

Using the same protocol, the deposition was investigated using a subphase containing $200 \mathrm{mM}$ ethanol, chosen since it produced a noticeable change in the shape of the isotherm. However the change was not as significant as that caused by 1-propanol, which altered both the position and shape of the isotherms. The dip profiles at each of the surface pressures tested are shown in figure 4.

When $200 \mathrm{mM}$ ethanol was incorporated into the subphase, similar behaviour was seen at low pressures, with irregular deposition and peeling off occurring. However at $35 \mathrm{mN} \mathrm{m}^{-1}$ the deposition became Y-type with good transfer ratios. Above this pressure the deposition was still regular but with apparent transfer ratios greater than $100 \%$ at $37.5 \mathrm{mN} \mathrm{m}^{-1}$ and more so at $40 \mathrm{mN}$ $\mathrm{m}^{-1}$. This is thought to be due to the monolayer stability decreasing as we approach the lowered collapse pressure. However when there is no deposition of film taking place the monolayer surface area rapidly stabilises, indicating that deposition can destabilise the monolayer to some extent.

\subsection{Behaviour of Res-2}

A second calix[4]resorcinarene was investigated with the aim of assuring that the behaviour demonstrated for Res- 1 is not unique to that material. This material was chosen because it displays a quite different structure, containing both sulphonate groups and long, dialkyl dimethyl 
ammonium counter-ions. Figure 5 shows the isotherms on water and $200 \mathrm{mM}$ ethanol. As was observed for Res-1, there is a noticeable decrease in both area per molecule and collapse pressure. Deposition profiles were also measured on water and $200 \mathrm{mM}$ ethanol subphases as before and the results shown in figure 6.

Again low surface pressure led to poor deposition. However, Res-2 deposited well at $40 \mathrm{mN} \mathrm{m}^{-1}$, although at this highest pressure there was again some slow collapse leading to apparent transfer ratios $>100 \%$. When ethanol was added to the subphase, the pressure required for consistent transfer fell to $35 \mathrm{mN} \mathrm{m}^{-1}$ and again there was a problem with collapse above this pressure as shown in the deposition trace (figure 6) at $37.5 \mathrm{mN} \mathrm{m}^{-1}$. Figure 6 shows a typical trace where there is a sudden loss of monolayer area at $37.5 \mathrm{mN} \mathrm{m}^{-1}$.

\subsection{Deposition onto optical fibres}

Due to the slow collapse of the Res-1 monolayer at higher pressures it was felt that in addition to simple transfer ratios the difference between water and alcohol doped subphases should be typified using a coated sensor system. The deposition of LB films onto optical fibres has been investigated previously, facilitating the demonstration of chemical sensors and non-linear optical effects $[18,20,21]$. In recent years there has been interest in the use of optical fibre long period gratings (LPGs) as a chemical sensing platform, where the grating facilitates interaction of light propagating in the optical fibre with a chemically sensitive overlayer, as given an appropriate combination of coating thickness and grating period the transmission spectrum of the optical fibre shows a high sensitivity to changes in the thickness and/or refractive index of the coating [22]. Here we use an LPG to facilitate the characterisation of the deposition of an LB film onto an optical fibre.

An LPG consists of a periodic modulation of the refractive index of the core of an optical fibre [23]. The period of the LPG, typically in the range $100 \mathrm{~nm}$ to $1 \mathrm{~mm}$, is such that light is coupled from the core of the fibre to a subset of symmetrical cladding modes at discrete wavelengths, creating attenuation bands within the transmission spectrum of the fibre, with the resonant wavelengths governed by the phase matching expression that is dependent upon the period of the grating and the effective refractive indices of the coupled modes [23]. The central wavelength of the resonance bands, by virtue of the dependence of the effective index cladding modes on the surrounding refractive index, show a sensitivity to the surrounding refractive index, and to the refractive index and thickness of a coating deposited onto the cladding, with high sensitivity for coating thicknesses of the order of $100 \mathrm{~nm}$ [22].

An LPG of period $97 \mu \mathrm{m}$ and length $35 \mathrm{~mm}$ was fabricated in hydrogen loaded single mode fibre of cut-off wavelength $650 \mathrm{~nm}$, SM750, via UV inscription using the point by point technique [20]. The transmission of the optical fibre devices were monitored in real time during the coating deposition process using an Ocean Optics (Geograaf 24, 6921 EW Duiven, Netherlands) S2000 spectrophotometer was used. The S2000 has a wavelength range of 200 to $1100 \mathrm{~nm}$ and a resolution of $\sim 0.3 \mathrm{~nm}$. The spectrometer was interfaced to a PC via a USB cable and the spectra recorded using Ocean Optics' software. 
In section 3.1 it was shown that Res- 1 coats irregularly at $30 \mathrm{mN} \mathrm{m}^{-1}$ on water at this surface pressure. It is not until higher surface pressures of $37.5 \mathrm{mN} \mathrm{m}^{-1}$ that Z-Type bilayer Langmuir Blodgett coating is achieved. This difference in the characteristics of LB coating should also be observable in the response of the transmission spectrum of an LPG to the deposition of a multilayer LB coating.

In order to demonstrate the apparent difference between subphase materials the $97 \mu \mathrm{m}$ taper was coated with Res- 1 at surface pressures of 30,35 and $37.5 \mathrm{mN} \mathrm{m}^{-1}$ spread on water and $200 \mathrm{mM}$ ethanol subphase; this range was chosen as it includes the point at which the coating changes from monolayer to bilayer.

Examples of the evolution of the LPG transmission spectrum during LB coating deposition are shown in Figure 7(a) and (b) for pure water subphases at $30 \mathrm{mN} \mathrm{m}^{-1}$ and $37.5 \mathrm{mN} \mathrm{m}^{-1}$ respectively. The white bands in the above grey scale plot show the movement of the resonance bands in the LPG transmission spectrum in response to increasing coating thickness. The figures illustrate a number of significant features of coated LPGs. Focusing on the resonance bands labelled LP016, LP017, and LP018 for number of coating bilayers between 20 and 30 the resonance wavelengths show high sensitivity to changes in the optical thickness of the coating. This region, termed the mode transition region, corresponds to the coating thickness at which one of the cladding modes is coupled into the coating, requiring a reorganization of the cladding modes, which causes significant changes in the cladding modes' refractive indices with concomitant changes in the resonance conditions. The second feature concerns the resonance band labelled LP019, which shows the development of a resonance band at a wavelength of approximately $790 \mathrm{~nm}$ for 56 layers. With increasing coating optical thickness the band splits into two. This effect is related to the properties of the phase matching condition, which contains a turning point.

Figure 7(a) and (b) show similar features. The onset of the phase matching turning point, the apex of the " $u$ " shaped feature, is a sensitive measure of the coating thickness. As the surface pressure increases, the position of the apex decreases in cycle number. This decrease is due the increase in the optical thickness of the coating with each cycle. On water it is noticeable that deposition at 35 $\mathrm{mN} \mathrm{m}^{-1}$ leads to a much less effective coating (in terms of optical thickness) than at $37.5 \mathrm{mN} \mathrm{m}^{-1}$. This change in thickness is most likely the result of the shift of the coating from irregular monolayer to bilayer and as is supported by the doubling in optical thickness of the coating when comparing 35 to $37.5 \mathrm{mN} \mathrm{m}^{-1}$. Table 1 summarises the results obtained when coating an LPG for different surface pressure for both pure water and $200 \mathrm{mM}$ ethanol subphases.

The apex positions show the same relationship of increasing coating thickness with an increase in surface pressure. The pure water coating shows no significant improvement in the coating of the sensor until the surface pressure reaches $37.5 \mathrm{mN} \mathrm{m}^{-1}$, whereas coating on a $200 \mathrm{mM}$ ethanol subphase shows some improvement at the lower $35 \mathrm{mN} \mathrm{m}^{-1}$ surface pressure and a further improvement on increasing the pressure to $37.5 \mathrm{mN} \mathrm{m}^{-1}$. This transition is indicative of the effect predicted by the isotherm changes and is an indicator of bilayer coating at lower surfaces pressures. 


\section{Conclusions}

The results presented here show both the strength of the interaction between monolayers and an aqueous subphase and the potential for modification of this interaction. This modification of the monolayer's properties through the addition of alcohol shows a clear trend, with a reduction in the dipole moment and in the hydrogen bonding potential. The large drops in collapse pressure in line with the reduced dipole-dipole interactions, especially for propan-1-ol, agrees with previous results showing that the collapse is dependent on the interaction of the monolayer with the water [4-6,8]. Changing the dipole and hydrogen bonding properties modifies the interaction of the monolayer material with the subphase and allows the release of the material at a lower surface

pressure. For both materials good quality Y-type deposition could be obtained at lower pressures on ethanol containing subphases than on pure water. In the case of other materials, using such additives could well allow the deposition of species, which so far, although forming stable monolayers, have been impossible to transfer to solid substrates from a pure water subphase.

We have also successfully used optical fibres modified long-period gratings to monitor deposition of monolayers onto the fibre surface and the effect of subphase modification on coating quality.

\section{Acknowledgments}

This work was supported by Leksing Ltd and the EPSRC grant no. EP/H02252X 


\section{References}

[1] D. Hoenig, D. J. Phys. Chem. 95 (1991) 4590.

[2] A. Tronin, J. Strzalka, V. Krishnan, I. Kuzmenko, H.C. Fry, M. Therien, J.K. Blasie, Rev. Sci. Instrum. 80 (2009) 033102.

[3] G. Richmond, G. Ann. Rev. Phys.Chem. 52 (2001) 357.

[4] G.L. Gaines, Insoluble monolayers at liquid-gas interfaces, John Wiley \& Sons Inc, New York, 1966.

[5] F. G. Moore, G. L. Richmond, Acc. Chem. Res. 41 (2008) 739.

[6] O.N. Oliveira, D.M. Taylor, T.J. Lewis, S. Salvagno, C.J.M. Stirling, J. Chem. Soc., Faraday Trans. 1, 85 (1989) 1009.

[7] S. Baoukina, L. Monticelli, H.J. Risselada, S. J. Marrink, D.P. Tieleman, Proc. Natl. Acad. Sc.i USA 105 (2008) 10803.

[8] E. Hatta, Langmuir, 20 (2004) 4059.

[9] M. Petty, Langmuir-Blodgett films: an introduction, Cambridge University Press, Cambridge, 1996.

[10] J. Kmetko, A. Datta, G. Evmenenko, P. Dutta, J. Phys. Chem. B, 105 (2001) 10818.

[11] R. Ghaskadvi, S. Carr, M. Dennin, J. Chem. Phys. 111 (1999) 3675.

[12] J. M. R. Patino, M. R. Dominguez, J. F. Feria, J. Coll. Inter. Sci. 154 (1992) 146.

[13] J. F. Feria, M. R. Dominguez, Langmuir 11 (1995) 2169.

[14] M. R. Dominguez, J. F. Feria, J. Amer. Oil. Chem. Sci. 76 (1999) 1041.

[15] J. M. R. Patino, J. F. Feria, C. G. Herrara, J. Coll. Inter. Sci. 154 (1992) 223.

[16] M. Weis, M. Kopani, J. Jakubovsky, E. Danihel, Appl. Surf. Sci. 253 (2006) 2425.

[17] M. Partridge, F. Davis, S.W. James, R.P. Tatam, C.F.J. Faul and S.P.J. Higson, Thin Solid Films, 520 (2012) 6989.

[18] S. Topliss, S.W. James, F. Davis, S.P.J. Higson, R.P. Tatam, Sens. Actuat. B, 143 (2010) 629.

[19] G. Vazquez, E. Alvarez, J. Chem. Eng. Data, 40 (1995) 611.

[21] D. Flannery, S. W. James, R. P. Tatam, G. J. Ashwell, Appl. Opt. 38 (1999) 7370.

[22] S. S. Johal, S. W. James, R. P. Tatam, G. J. Ashwell, Opt. Lett. 24 (1999) 1194. 
[23] S. W. James, R. P. Tatam, J. Opt. A: Pure and Applied Optics, 8 (2006) S430.

[24] S. W. James, R. P. Tatam, Measure. Sci. Tech. 14 (2003) R49. 
List of tables and figures.

Figure 1. Structures of the monolayer materials used in this work.

Figure 2. The dipping head showing how the fibre was mounted.

Figure 3. Res-1 isotherms on subphase containing different additives (200 $\mathrm{mM})$ to the subphase.

Figure 4. Deposition profiles for Res-1 on water and $200 \mathrm{mM}$ ethanol

Figure 5. Res-2 isotherms on water and $200 \mathrm{mM}$ ethanol.

Figure 6. Deposition profiles for Res-2 on water and $200 \mathrm{mM}$ ethanol.

Figure 7. Grey scale plots of the coating with Res-1 at surface pressures of 35 and $37.5 \mathrm{mN} \mathrm{m}-1$ on a water subphase over 200 cycles (only the 'air' traces are shown).

Table 1. Table of coupling location on two different subphases 
Table 1.

Table 1: Table of coupling location (marked on Figure 7a and b) on two different subphases.

\begin{tabular}{|l|l|l|}
\hline $\begin{array}{l}\text { Coating surface } \\
\text { pressure }\left(\mathrm{mN} \mathrm{m}^{-1}\right)\end{array}$ & $\begin{array}{l}\text { Apex position (cycle number) with } \\
\text { pure water subphase }\end{array}$ & $\begin{array}{l}\text { Apex position (cycle number) with } \\
200 \mathrm{mM} \text { ethanol subphase }\end{array}$ \\
\hline 30 & 94 & 92 \\
\hline 35 & 90 & 69 \\
\hline 37.5 & 43 & 49 \\
\hline
\end{tabular}


Figure 1

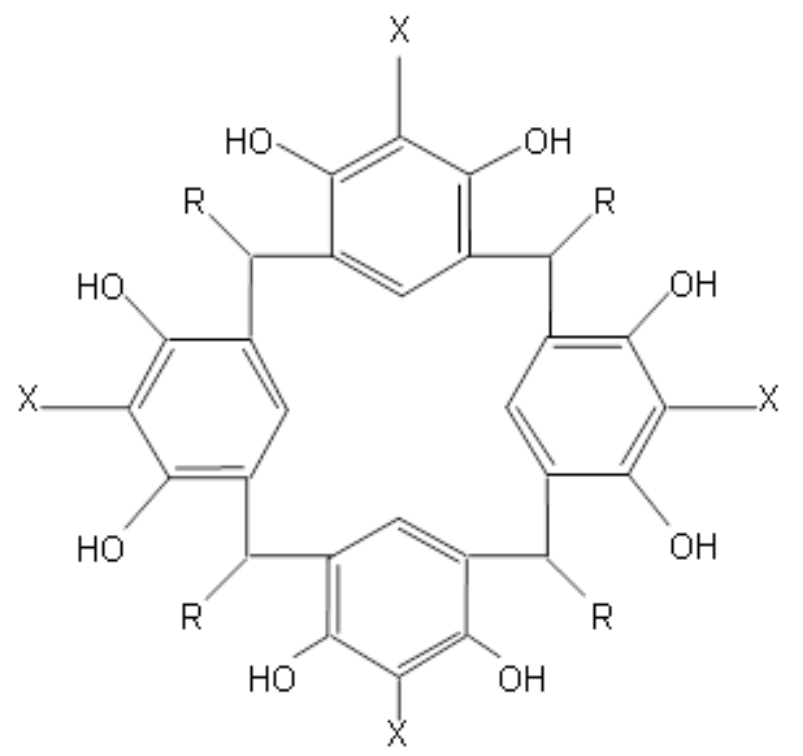

$$
\begin{array}{lll}
\text { Res-1 } & \mathrm{R}=\mathrm{C}_{11} \mathrm{H}_{23} & \mathrm{X}=\mathrm{H} \\
\text { Res-2 } & \mathrm{R}=\mathrm{C}_{11} \mathrm{H}_{23} & \mathrm{X}=\mathrm{CH}_{2} \mathrm{SO}_{3}-\left(\mathrm{C}_{16} \mathrm{H}_{33}\right)_{2} \mathrm{~N}^{+}\left(\mathrm{CH}_{3}\right)_{2}
\end{array}
$$

Figure 2.

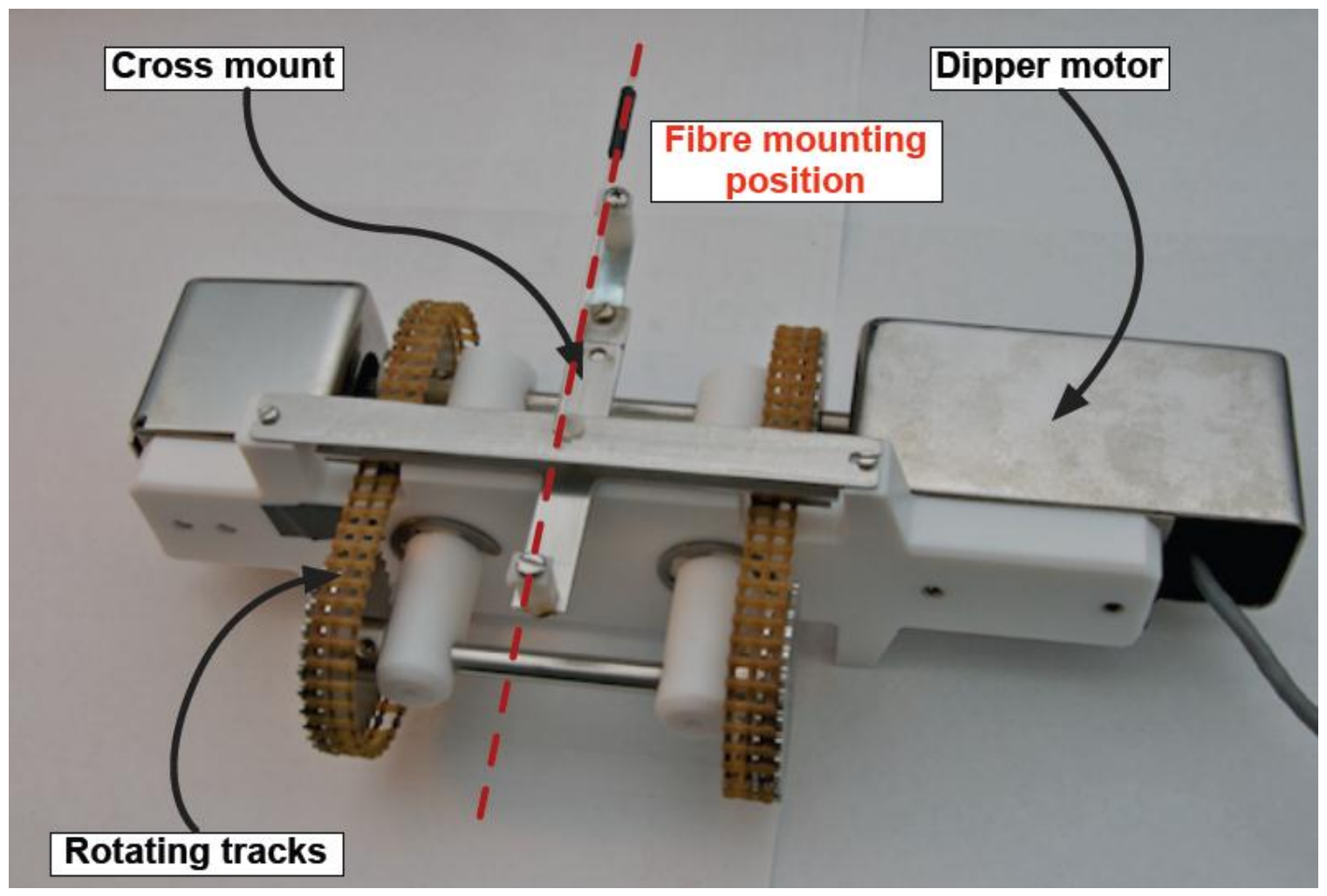


Figure 3.

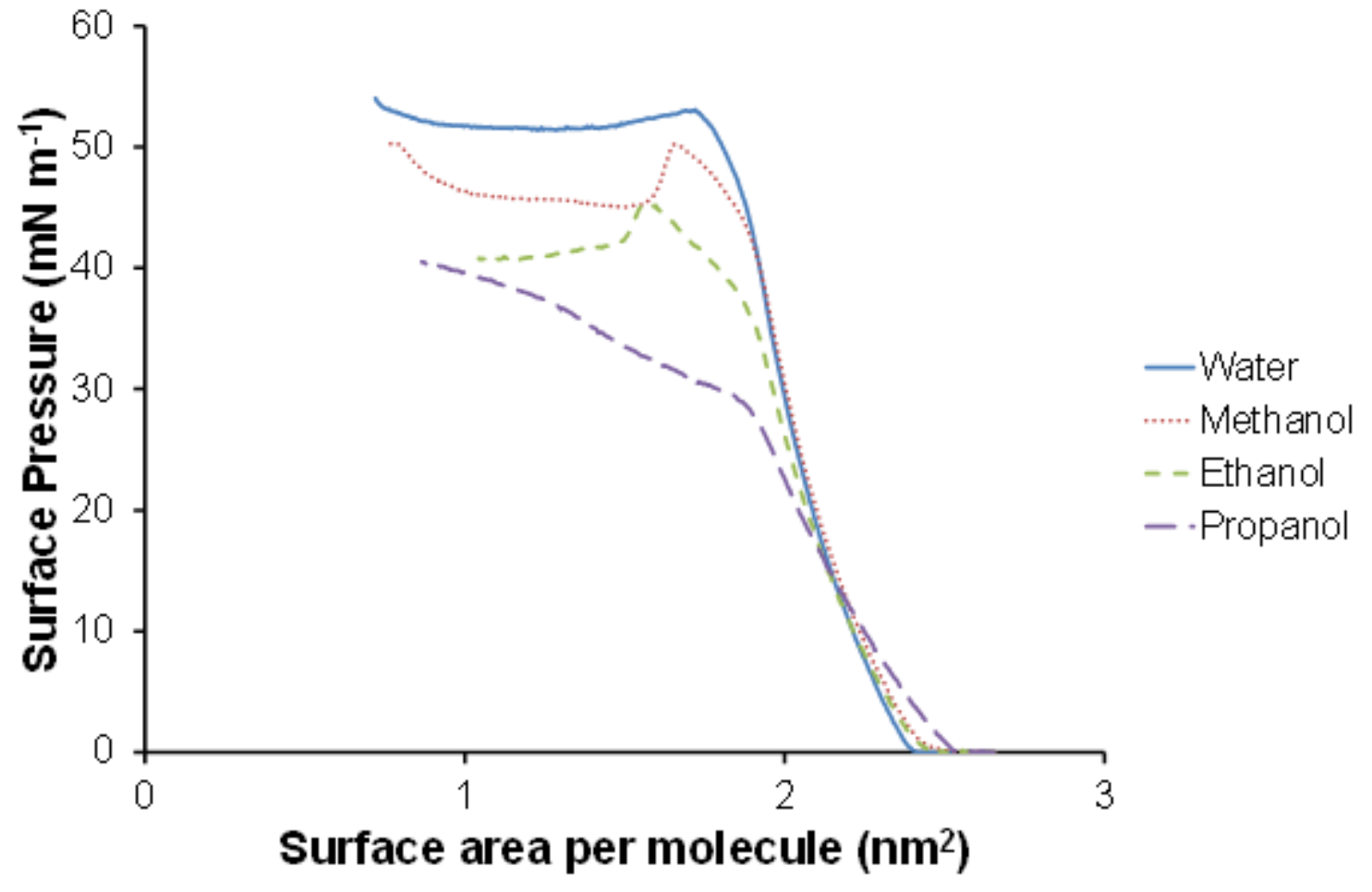


Figure 4.

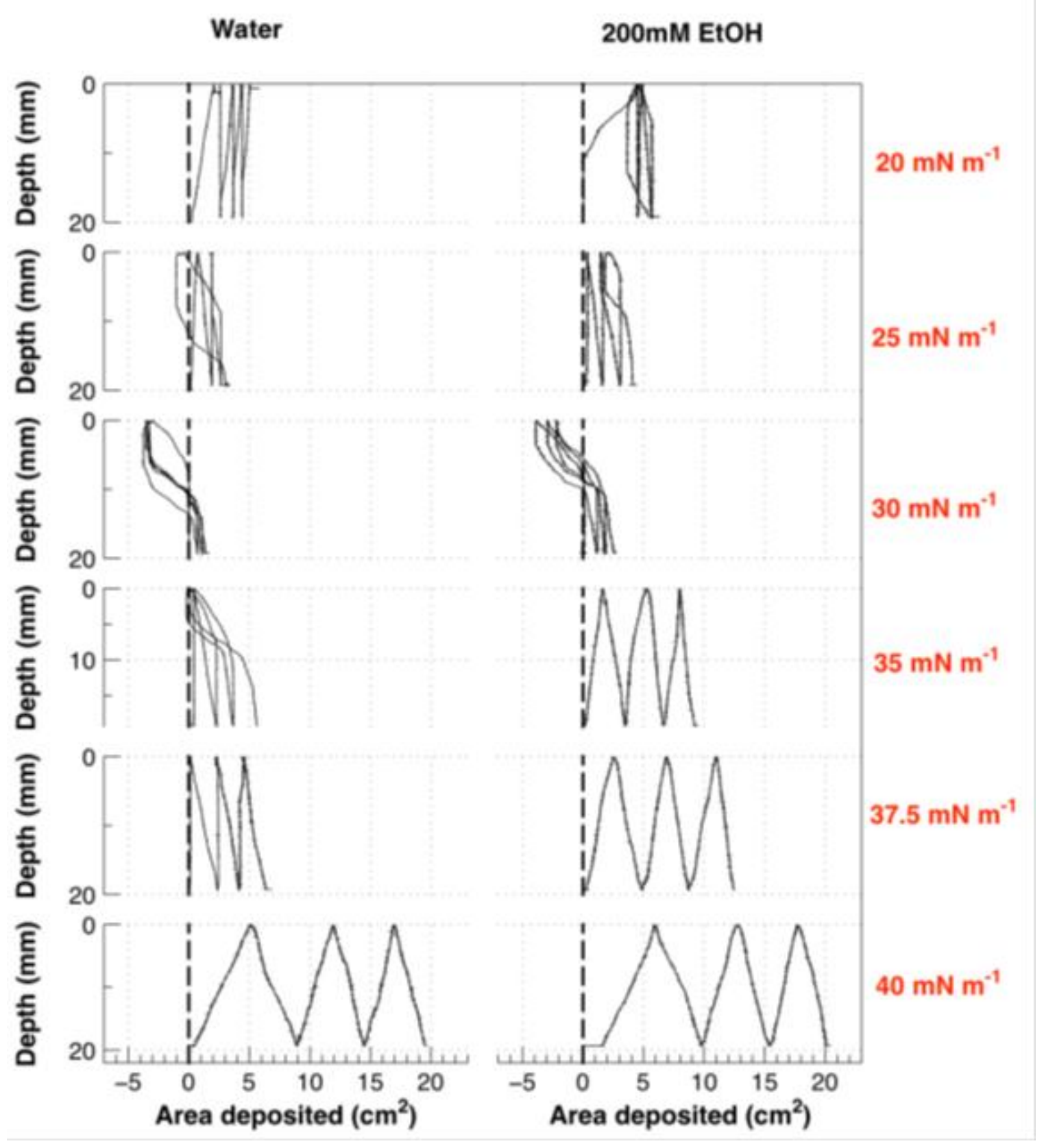


Figure 5.

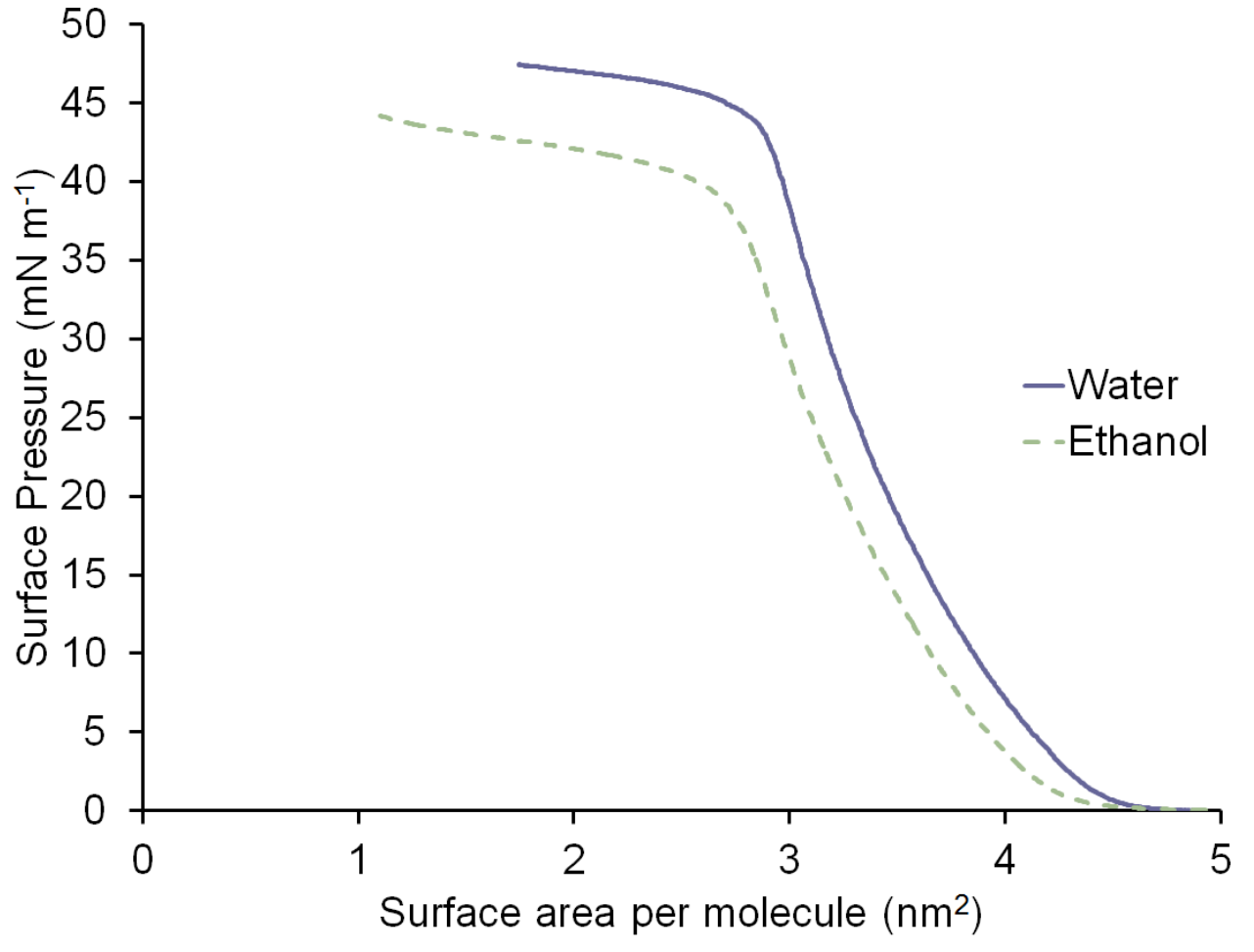


Figure 6.

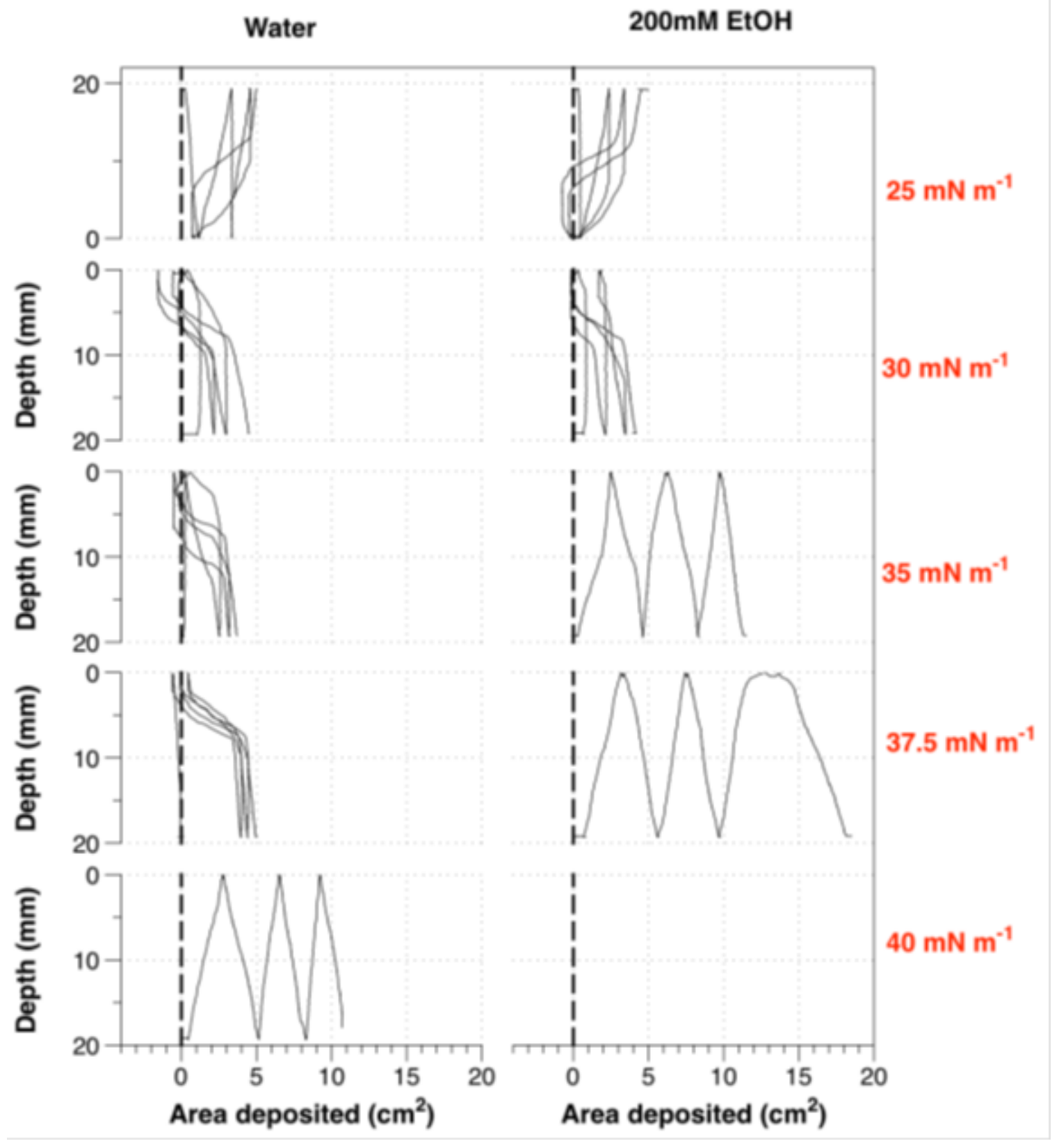


Figure 7.
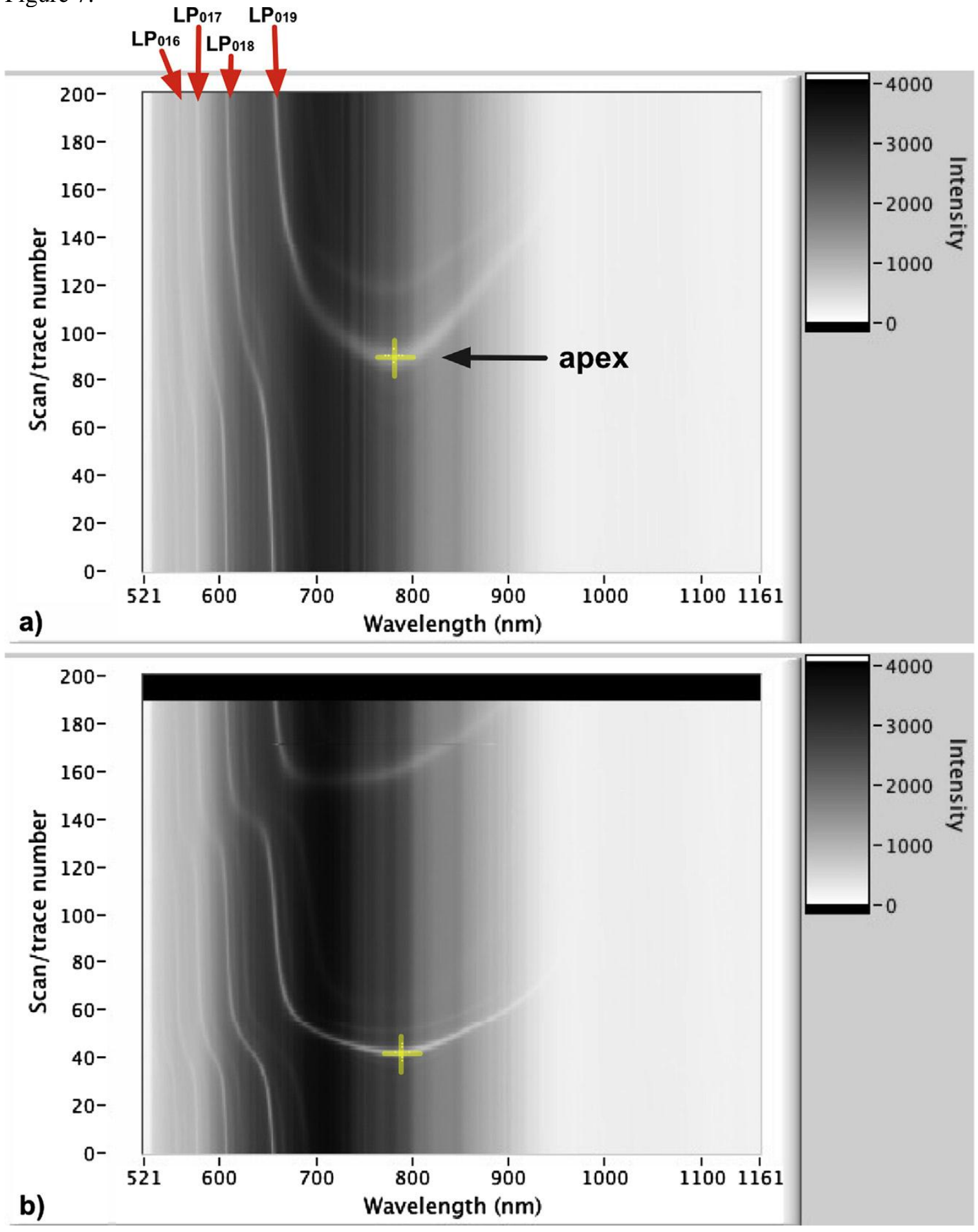
2014-01-17

Modifying monolayer behaviour by

incorporating subphase additives and

pÿimproving Langmuir Blodgett thin film deposition on optical fibres

\section{Partridge, Matthew}

\section{Elsevier}

Matthew Partridge, Rebecca Wong, Mike Collins, et al., Modifying monolayer behaviour by pÿincorporating subphase additives and improving Langmuir Blodgett thin film deposition on pÿoptical fibres. Materials Chemistry and Physics, Volume 144, Issues 12, 14 March 2014, pp. 179-185 http://dx.doi.org/10.1016/j.matchemphys.2013.12.040.

Downloaded from Cranfield Library Services E-Repository 